\title{
Spadin, a Sortilin-derived peptide: a new concept in the antidepressant drug design
}

\author{
Catherine HEURTEAUX \\ Jean MAZELLA \\ Marc BORSOTTO
}

Institut de pharmacologie moléculaire et cellulaire, CNRS, Université de Nice Sophia Antipolis, 660 Route des Lucioles, 06560 Valbonne, France $<$ heurteaux@ipmc.cnrs.fr $>$

\begin{abstract}
Depression is the most common of psychiatric illnesses. The design of effective treatments for this disorder is a challenging process. Recently, the two-pore domain potassium channel TREK-1 has been identified as a new target in depression, and its antagonists might become effective antidepressants. Deletion of TREK-1 gene results in a depression-resistant phenotype that mimics antidepressant treatments. Here, we validate the fast antidepressant effects of spadin, a secreted peptide derived from the propeptide generated by the maturation of the sortilin receptor and acting through TREK-1 inhibition.
\end{abstract}

Key words: Spadin, TREK-1 channel, depression, behavior
Depression is a devastating illness that affects $\sim 17 \%$ of the population at some point in life, resulting in major social and economic consequences (Kessler et al., 2005). The use of antidepressants has an overall low clinical efficacy as full remission only occurs in one-third of the patients. In the case of response, side effects are often observed, as well as a delay in the onset of therapeutic efficiency. Most antidepressants increase levels of the monoamine serotonin (5-HT) and/or noradrenaline (NA), suggesting that biochemical imbalances within the 5-HT/NA systems may underlie the pathogenesis of this disorder (Nestler et al., 2002; Krishnan and Nestler, 2008). To date, the mainstay of antidepressant treatments is constituted by selective serotonin reuptake inhibitors, which inhibit the 5 -HT reuptake pump. Improving the treatment of depression is challenging. Recently, mouse models of depression have highlighted the putative role of the TREK-1 channel in the mechanisms of action of antidepressants. The TREK-1 channel (figure 1A) belongs to the particular family of two-pore-domain potassium channels (Lesage and Lazdunski, 2000). It is open at membrane potentials in physiological conditions and contributes to the background or leak currents that set the resting potential and oppose depolarizing influences. TREK-1 is activated by volatile general anesthetics, membrane stretch, heat, acidosis, but also by riluzole, polyunsaturated fatty acids and lysophospholipids (Lesage and Lazdunski, 2000; Honore, 2007). These openers prevent neuronal death in animal models of epilepsy, stroke, global, retinal and spinal ischemia (Blondeau et al., 2002a,b; Blondeau et al., 2007; Ettaiche et al., 1999; Heurteaux et al., 2006a; LangLazdunski et al., 1999, 2003; Lauritzen et al., 2000). The phenotyping of TREK1 deficient mice $\left(\mathrm{kcnk}^{-/-}\right)$has identified a major role for TREK1 channels in the control of epileptogenesis, general anesthesia, polyunsaturated fatty acidsinduced neuroprotection (Heurteaux et al., 2004) and pain (Alloui et al., 2006; Noel et al., 2009). The TREK-1 channel is physiologically blocked by phosphorylation processes such as phosphorylation resulting from $\mathrm{Gs}$ or Gq Gproteins. It is also inhibited by SSRIs such as fluoxetine (Heurteaux et al., 2006). Deletion of the TREK-1 gene (also called kcnk2) results in a depression-resistant phenotype that mimics treatment with antidepressants (Heurteaux et al., 2006). Interestingly, the Star*D study has identified an association between the existence of four genetic variants (SNPS) in the TREK-1 locus, and a resistance to multiple antidepressant classes (Perlis et al., 2008; Dillon et al., 2010). All these findings indicate that 1) genetic variations in TREK-1 may identify individuals at risk for depression treatment resistance and 2) a search of selective blockers of TREK-1, hitherto not available, might potentially lead to a new generation of antidepressants.

Growing evidence indicates that trafficking and addressing as well as functional properties of native ion channels depend on their lipidic and proteic environments. $\mathrm{K}^{+}$channels are known to interact with partner proteins that are crucial for their regulation. In search of protein partners, we identified the sortilin receptor (Mazella et al., 1998; Nielsen et al., 2001) in the regulation of the channel function (figure 1B). Sortilin is a 95-100kDa type-1 membrane protein, consisting of a large luminal domain, a single transmembrane segment and a short C-terminal cytoplasmic tail. A large part of sortilin is localized at the level of the 


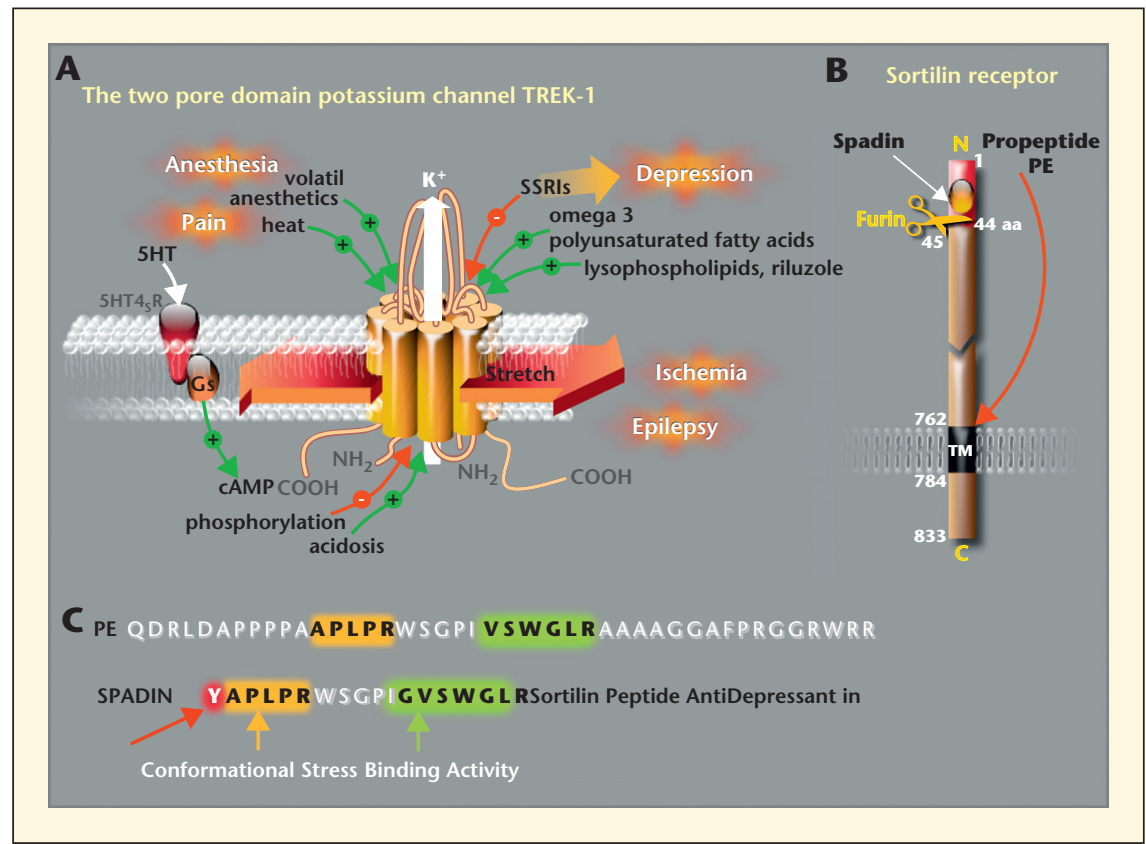

Figure 1. The two pore domain potassium channel TREK1 and the sortilin receptor:

A) Activation and Inhibition of TREK-1 and involvement of TREK-1 in cerebral pathologies ;

B) Cleavage of the mature ligand-binding receptor by furin and release of the propeptide (PE).

C) Identification of spadin sequence.

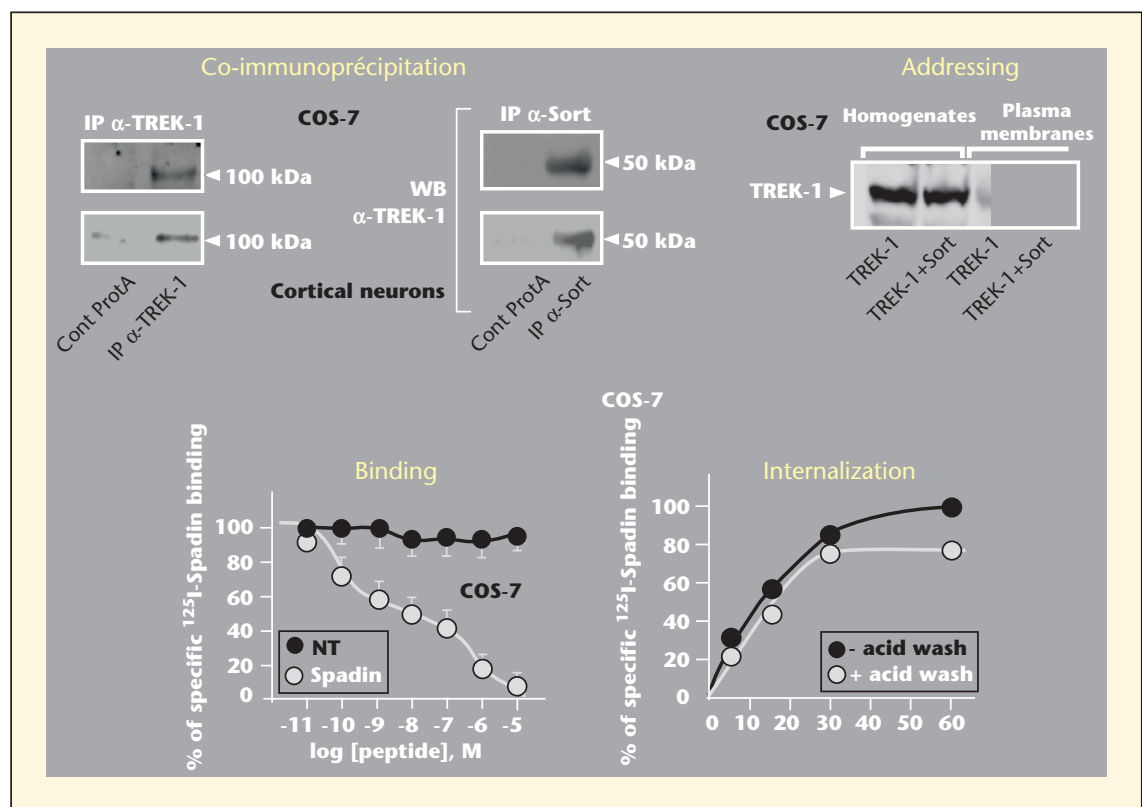

Figure 2. Sortilin and Spadin interact with the TREK-1 channel. Co-immunoprecipitation: Immunoprecipitation of Sortilin with anti-TREK-1 antibody (IP $\alpha$-TREK-1) or immunoprecipitation of TREK-1 with anti- Sortilin antibody (IP $\alpha$-Sort) from transfected COS-7 cells or mouse cortical neurons. Immunoprecipitated proteins are subjected to Western blots and revealed using anti-sortilin (WB: $\alpha$-Sort) or anti-TREK-1 (WB: $\alpha$-TREK-1). Addressing: Influence of Sortilin on the expression of TREK-1 at the plasma membranes. COS-7 cells are transfected with TREK-1 in the absence or in the presence of Sortilin. Crude homogenates, purified plasma membrane proteins or cell surface biotinylated proteins are subjected to Western blot analysis and revealed using anti-TREK-1 antibody. Binding: Competition between ${ }^{125}$ I-Spadin and unlabeled Spadin (white circles) or NT (black circles) for binding to TREK-1 transfected COS-7 cell homogenates.
Golgi apparatus where the protein triggers intracellular functions of trafficking. Depending on its cellular location, sortilin may also act as a receptor or a coreceptor and binds neurotensin (NT), the precursor of the Nerve Growth Factor (proNGF), the receptor-associated protein (RAP), the lipoprotein lipase and the propeptide released from its precursor form (Mazella et al., 1998; Nykjaer et al., 2004). Sortilin as well as TREK-1 are highly expressed in cerebral structures involved in the pathophysiology of depression, such as prefrontal and cingulate cortice, amygdala, hippocampus, nucleus accumbens, dorsal raphe and hypothalamus. Sortilin is synthesized as a proform (prosortilin) which, in late Golgi compartments, is converted to the functional ligand-binding receptor by cleavage and release of a 44 residue $\mathrm{N}$ terminal propeptide (Gln ${ }^{1}-\mathrm{Arg}^{44}$, propeptide) by furin (Munck Petersen et al., 1999). Propeptide binds to the mature receptor with a high affinity $(\mathrm{Kd} \sim$ $5 \mathrm{nM})$. Structure-function relationship studies have identified that the peptide $\mathrm{GIn}{ }^{1}-\mathrm{Arg}^{28}$ was as efficient on the binding activity as the entire propeptide GIn ${ }^{1}-\mathrm{Arg}^{44}$, whereas the affinity of the peptide Gln ${ }^{1}-$ Arg $^{16}$ was very low (Westergaard et al., 2004) (figure 1C). Therefore, we designed the peptide spadin by conserving the sequence 1728 in which we added the sequence 1216 (APLRP) in order to maintain conformational stress. This partial propeptide (Ala $\left.{ }^{12}-\mathrm{Arg}^{28}\right)$ called spadin (figure 1C) was tested for its potential effects on TREK-1 channel regulation and for its validation as antidepressant drug in five behavioral models of depression (Mazella et al., 2010).

The first result of this work is the identification of the NTSR3/Sortilin receptor as a novel TREK-1 partner protein (figure 2). It interacts physically and functionally with TREK-1 to modify its cell surface expression. Experiments of immunoprecipitation of TREK-1 and Sortilin showed that each antibody immunoprecipitated the tested partner, i.e. sortilin precipitated with the TREK-1 antiserum, and TREK- 1 with the anti-

Internalization, Association kinetics of 125 . Spadin binding to COS-7 cells tranfected with TREK-1. At the indicated times, cells are either washed twice with $500 \mu \mathrm{L}$ of binding buffer (black circles) or treated with $500 \mu \mathrm{L}$ of acid$\mathrm{NaCl}$ buffer for $2 \mathrm{~min}$ (white circles). 


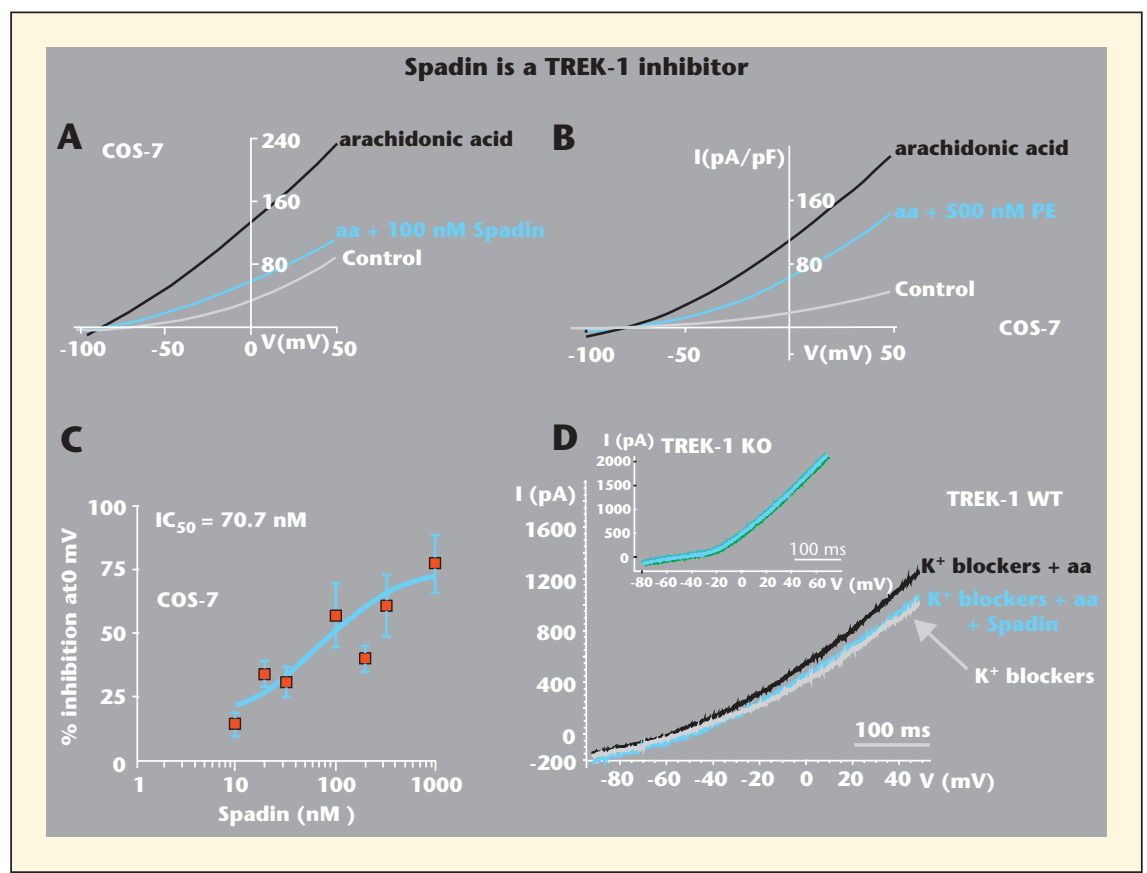

Figure 3. Effects of Spadin on the TREK-1 channel activity. A-B) Whole-cell currents measured in COS-7 transfected cells in presence of potassium blockers ( $K^{+}$blockers, $10 \mathrm{mM}$ tetraethyl ammonium (TEA), $3 \mathrm{mM}$ 4-aminopyridine (4-AP), $50 \mathrm{nM}$ charybdotoxin, $10 \mu \mathrm{M}$ glibenclamide, $100 \mathrm{nM}$ apamin). Cells are clamped at $-80 \mathrm{mV}$ and voltage changes are applied by ramp from -100 to $50 \mathrm{mV}, 1 \mathrm{~s}$ in duration Currents are recorded after TREK-1 activation by $10 \mu \mathrm{M}$ arachidonic acid (aa) and aa + Spadin (100 nM) (A) or aa + propeptide (PE, $500 \mathrm{nM})$ (B). Native currents are recorded in the absence (Control) and in the presence of spadin or PE.

C) Dose-dependent spadin inhibition of TREK-1 currents, IC $C_{50}$ value at $0 \mathrm{mV}$ is of $70.7 \mathrm{nM}$. Currents are measured in the presence of $10 \mu \mathrm{M}$ aa. D, Native currents recorded in the presence of $K^{+}$blockers after stimulation by $10 \mu \mathrm{M}$ of aa on CA3 pyramidal neurons from hippocampus slices in wild-type mice (D) or in kcnk2 deficient mice (TREK-1 KO) (Inset) in the presence or the absence of spadin $(1 \mu \mathrm{M})$. Currents are elicited by a ramp from $-100 \mathrm{mV}$ to $50 \mathrm{mV}$.

sortilin antibody (figure 2 Co-immunoprecipitation), in both COS-7 cells and cortical neurons co-expressing both proteins. The expression of TREK-1 within the plasma membranes, measured either by preparing purified plasma membranes or by using cell surface biotinylation, was enhanced (by a factor 3 and 6, respectively) when COS-7 cells were cotransfected with sortilin (figure 2 Addressing), confirming the interaction between the two proteins, at least during the channel sorting. Spadin displays identical binding and functional properties as those of the full length propeptide on the neurotensin system. Competition experiments between ${ }^{125}$-labelled spadin and unlabelled spadin or NT on membrane homogenates from TREK-1 transfected COS-7 cells showed that spadin bound specifically to TREK- 1 with an affinity of about $10 \mathrm{nM}$. This binding was selective arachidonic acid (aa, $10 \mu \mathrm{M}$ ), which induced a typical TREK-1 background current, characterized by outward rectification reversed at the predicted value for $E_{K_{+}}$. Using the whole-cell patchclamp technique on TREK-1 transfected COS-7 cells, we demonstrated that 100 nM of spadin were able to block 63 $\pm 12 \%$ of the TREK-1 current stimulated by aa (figure $3 A$ ). A spadin doseresponse experiment indicated an $\mathrm{IC}_{50}$ value of $70.7 \mathrm{nM}$ at $0 \mathrm{mV}$ (figure 3C). Spadin displayed a better affinity than the propeptide, since $500 \mathrm{nM}$ of propeptide were necessary to block $41 \pm 5 \%$ of the aa stimulated TREK-1 current measured at $0 \mathrm{mV}$ (figure $3 B$ ). Spadin also blocked the TREK-1 activity in CA3 hippocampal neurons on brain slices of wild-type mice and not in $\mathrm{kcnk}^{-/-}$mice, suggesting a specific inhibitory effect of spadin on the TREK-1 channel (figure 3D).

Finally, we point out spadin as the first peptidic and fast-acting antidepressant. Spadin efficacy was first assessed in the forced swimming test (FST), which is a highly reliable predictor for antidepressant potential (Krishnan and Nestler, 2008). Spadin was administered $30 \mathrm{~min}$ before the test by intracerebroventricular (i.c.v.), intravenous (i.v.) or intraperitoneal (i.p.) route at doses of $10^{-4}$ to $10^{-8} \mathrm{M}$. When placed in an inescapable cylinder of water, spadin-treated mice exhibited reduced floating or immobility times in the three modes of injection with respect to their saline-treated counterparts (figure $4 A$ ). The immobility is interpreted as "a state of despair". The doseresponses of spadin showed that the highest reduced immobility times were observed at the dose of $10^{-7} \mathrm{M}$ in i.c.v. $(66.8 \%), 10^{-6} \mathrm{M}$ in i.v. (62.9\%) and $10^{-5} \mathrm{M}$ in i.p. (55.30\%) administration. The magnitude of the antidepressant behavior was similar to that observed in fluoxetine-treated wild-type and salineinjected $\mathrm{kcnk}^{-/-}$mice. It has been proposed that a direct facilitation of 5-HT firing rate in the dorsal raphe nucleus (DRN) should be a requirement for a faster onset of antidepressant action (Blier, 2001). Interestingly, in $\mathrm{kcnk} 2^{-1-}$ mice we observed an increase in the firing activity of DRN 5-HT neurons (Heurteaux et al., 2006). Obviously, such results raise the possibility that spadin could exert a rapid onset of action. A 4-day treatment with spadin (i.v., $10^{-6} \mathrm{M}$ ) significantly reduced the time spent immobile by 


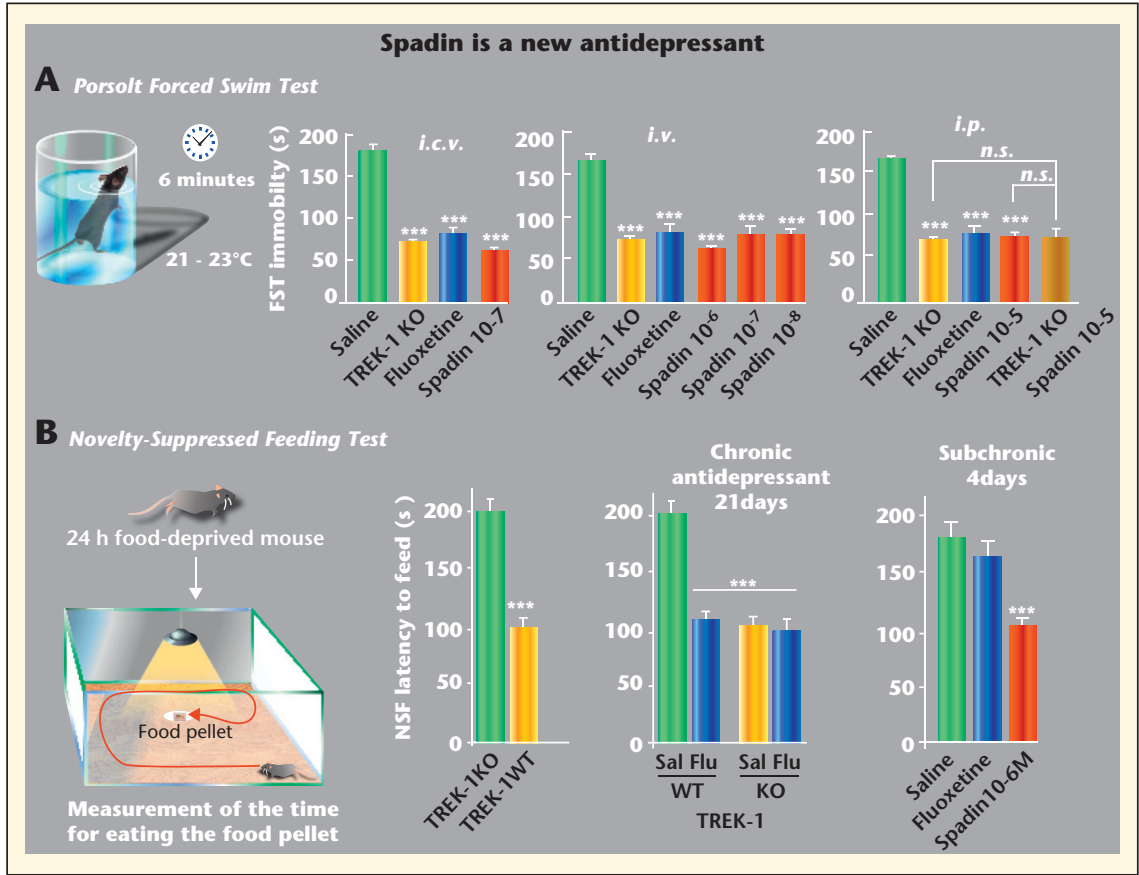

Figure 4. Antidepressant effects of Spadin. A) Forced Smimming Test (FST), spadin-treated mice have a shorter time of immobility comparable to that obtained with TREK-1 KO or fluoxetinetreated mice, whatever the way of spadin administration: intracerebroventricular (i.c.v), intravenous (i.v) or intraperitoneal (i.p). Acute treatments: Spadin $\left(10^{-4}\right.$ to $10^{-8} \mathrm{M}$ ) or Fluoxetine $(3 \mathrm{mg} / \mathrm{kg})$ or Saline solutions are injected $30 \mathrm{~min}$ before the test in wild-type and TREK-1 KO mice. B) Novelty-Suppressed Feeding Test (NSF): Subchronic treatments: Spadin $\left(10^{-6} \mathrm{M}\right)$, Fluoxetine $(1 \mathrm{mg} / \mathrm{kg})$ or Saline solutions are i.v. injected in a $100 \mu \mathrm{L}$ bolus once a day for 4 successive days before the test. In chronic treatments, spadin $\left(10^{-6} \mathrm{M}\right)$ and fluoxetine $(1 \mathrm{mg} / \mathrm{kg})$ are i.v. injected in a $100 \mu \mathrm{L}$ bolus once a day for 21 successive days. At the end of the $A D$ treatment, animals are food deprived for one day and then measured for their latency to feed. 4-day treatment with Spadin significantly reduces the latency to feed when compared to saline or fluoxetine treatments.

43.2\% in FST (data not shown). In contrast, subchronic fluoxetine treatment had no effect when compared with saline (data not shown).. The Novelty Suppressed Feeding test (NSF) is usually carried out for demonstrating antidepressant efficacy after chronic, but not acute treatment (Santarelli et al., 2003). Mice treated with spadin (i.v., $10^{-6} \mathrm{M}$ ) for 4 days showed a significant decrease in latency to feed relative to saline-injected animals (figure $4 B$ ). A 4-day regimen with fluoxetine (i.p., $3 \mathrm{mg} / \mathrm{kg}$ ) had no effect in the same conditions (figure 4B). In comparison, a chronic antidepressant treatment with fluoxetine during 21 days in wild-type and $\mathrm{kcnk}^{-/-}$mice significantly reduced the latency to feed as compared to saline (figure $4 B$ ).

The fast-acting antidepressant potential of spadin, observed in FST tests is further
Interestingly, a 4-day treatment with spadin (i.p. $10^{-5} \mathrm{M}$ ) significantly increased by two-fold the number of BrdU-positive cells with respect to saline conditions (figure 5A). In contrast, a 4day regimen with fluoxetine had no effect on neurogenesis, but fluoxetine induced a significant increase in the number of BrdU-positive cells when it was administered during 15 days (data not shown). Dual labeling of BrdU and doublecortin (DCX), a specific marker of neuronal precursors revealed that $85.2 \%$ of BrdU-labelled cells expressed DCX (data not shown).

As described in $\mathrm{kcnk} 2^{-/-}$mice (Heurteaux et al., 2006), spadin leads to an in vivo increase in efficacy of 5-HT neurotransmission as evidenced by an increased firing activity of DRN 5-HT neurons (figure 5C). The facilitation of central 5-HT transmission constitutes the common property of all the antidepressant strategies. From a mechanical point of view, $5-\mathrm{HT}_{1 \mathrm{~A}}$ autoreceptor stimulation reduces DRN 5-HT neuronal firing and, consequently, 5-HT neurotransmission (Blier and de Montigny, 1999). Inhibition of adenylate cyclase and activation of G-protein-coupled inwardly rectifying $\mathrm{K}+$ channels (GIRK) are involved in this negative feedback. The decrease in cAMP concentration (as a result of reduced adenylate cyclase activity) in 5-HT neurons is also thought to induce TREK-1 opening because of a consequent reduction of phosphorylation of Ser333 by PKA (Maingret et al., 2000). According to this model, spadin would induce a depolarization by closing TREK-1 channels and, as described for TREK-1 deficient mice would therefore reduce the negative feedback on 5-HT neurons, resulting in increased 5-HT neurotransmission and in turn in antidepressant-like effects. Direct inhibition of TREK-1 by spadin may also contribute to enhanced $5-\mathrm{HT}$ neuron excitability. Because (i) sortilin is the partner protein of the TREK-1 channel and (ii) both proteins are colocalized in 5-HT-enriched areas known to be involved in the pathophysiology of depression, one may infer that spadin acts predominantly through a modulation of the brain 5-HT circuitry. Nevertheless, we cannot exlude that it can also involve other neurotransmission systems. Whatever the effector pathways involved, the fact that spadin has no effects on $\mathrm{kcnk} 2^{-/-}$mice indicates that 


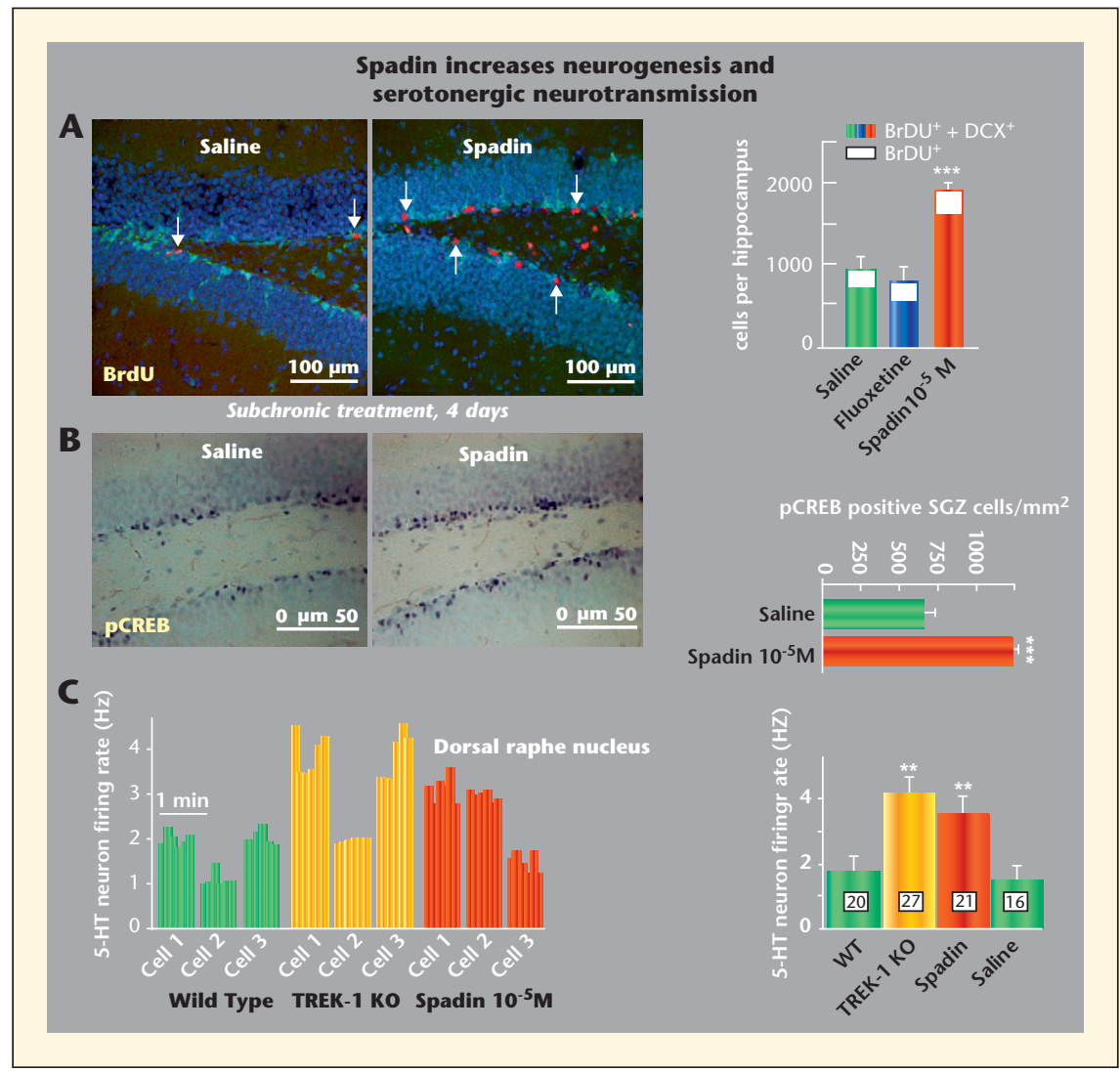

Figure 5. Effects of Spadin on neurogenesis, CREB activation and Serotoninergic neurotransmission. A) Spadin increases neurogenesis: (left), Representative photomicrographs of BrdU-labeled neurons in the dentate gyrus of the mouse hippocampus treated either with saline or with spadin (i.v., $10^{-6} \mathrm{M}$ ) for 4 days. (Right) Quantitation of BrdU positive cells of hippocampus treated with saline, fluoxetine or spadin $\left(10^{-5} \mathrm{M}\right)$ for 4 days. $85 \%$ of BrdU-labeled cells are positive to $D C X . B)$ Enhanced spadin treatment-induced CREB activation in the hippocampus, as assessed by measuring phosphoCREB (PCREB) immunoreactivity. (left), Immunological distribution of $p C R E B$ in the mouse hippocampus after a 4-day i.v treatment. $p C R E B$ is phosphorylated in the cells near the subgranular zone (SGZ). Right: Quantification of pCREB positive cells $/ \mathrm{mm}^{2}$ in hippocampal SGZ. C) Effect of spadin on the firing rate of DRN 5-HT neurons. Spadin ( $10^{-5} \mathrm{M}$ in a $100 \mu \mathrm{L}$ bolus) or its vehicle is i.p. administered. Recordings start $30 \mathrm{~min}$ after the injection, and are performed for a maximal duration of $210 \mathrm{~min}$ thereafter. Left: Samples of "descents" performed along the DRN, showing typical integrated firing rate histograms in a vehicle-, TREK-1 KO- or spadin-treated animal. Right: 5-HT neuron firing activity, calculated on the basis of all the cells recorded within the successive tracks performed along the DRN.

its action is first and foremost mediated by a modulation of TREK- 1 channels.

\section{Conclusion}

Spadin can be considered as a natural endogenous antidepressant and constitutes the first peptide identified as an antidepressant with a rapid onset of action. Due to these peculiar properties, spadin brings a new concept to address the treatment of depression. To date, spadin is also the first blocker
Acknowledgements. This work was supported by the Centre National de la Recherche Scientifique (CNRS), the Agence Nationale de la Recherche (ANR) and the Fondation pour la Recherche Médicale (FRM).

\section{REFERENCES}

Alloui A, Zimmermann K, Mamet J, et al. TREK-1, a K+ channel involved in polymodal pain perception. EMBO / 2006; 25: 2368-76.

Blier $\mathrm{P}$, de Montigny $\mathrm{C}$. Serotonin and druginduced therapeutic responses in major depression, obsessive-compulsive and panic disorders. Neuropsychopharmacology 1999; 21: 91S-98S.

Blier P. Possible neurobiological mechanisms underlying faster onset of antidepressant action. J Clin Psychiatry 2001; 62 (Suppl. 4): 7-11; discussion 37-40.

Blondeau N, Lauritzen I, Widmann C, Lazdunski M, Heurteaux C. A potent protective role of lysophospholipids against global cerebral ischemia and glutamate excitotoxicity in neuronal cultures. / Cereb Blood Flow Metab 2002; 22: 821-34.

Blondeau N, Widmann C, Lazdunski M, Heurteaux C. Polyunsaturated fatty acids induce ischemic and epileptic tolerance. Neuroscience 2002; 109: 231-41.

Dillon DG, Bogdan R, Fagerness J, Holmes AJ, Perlis RH, Pizzagalli DA. Variation in TREK1 gene linked to depression-resistant phenotype is associated with potentiated neural responses to rewards in humans. Hum Brain Map 2010; 31: 210-21.

Ettaiche M, Fillacier K, Widmann C, Heurteaux C, Lazdunski M. Riluzole improves functional recovery after ischemia in the rat retina. Invest Ophthalmol Vis Sci 1999; 40: 729-36.

Heurteaux C, Guy N, Laigle C, et al. TREK-1, a $\mathrm{K}(+)$ channel involved in neuroprotection and general anesthesia. EMBO / 2004; 23: 2684-95.

Heurteaux C, Lucas G, Guy N, et al. Deletion of TREK-1, a background potassium channel, results in a depression-resistant phenotype. Nature Neurosci 2006; 9: 1134-41.

Honore E. The neuronal background K2P channels: focus on TREK1. Nat Rev Neurosci 2007; 8: 251-61.

Kessler RC, Berglund P, Demler O, et al. Lifetime prevalence and age-of-onset distributions of DSM-IV disorders in the National Comorbidity Survey Replication. Arch Gen Psychiatry 2005; 62: 593-602.

Krishnan V, Nestler EJ. The molecular neurobiology of depression. Nature 2008; 455: 894-902. 
Lang-Lazdunski L, Blondeau N, Jarretou G, Lazdunski M, Heurteaux C. Linolenic acid prevents neuronal cell death and paraplegia after transient spinal cord ischemia in rats. I Vasc Surg 2003; 38: 564-75.

Lang-Lazdunski L, Heurteaux C, Vaillant N, Widmann C, Lazdunski M. Riluzole prevents ischemic spinal cord injury caused by aortic crossclamping. I Thorac Cardiovasc Surg 1999; 117: 881-9.

Lauritzen I, Blondeau N, Heurteaux C, Widmann C, Romey G, Lazdunski M. Polyunsaturated fatty acids are potent neuroprotectors. EMBO / 2000; 19: 1784-93.

Lesage F, Lazdunski M. Molecular and functional properties of two pore domain potassium channels. Am J Physiol 2000; 279: 793801.

Maingret F, Lauritzen I, Patel AJ, et al. TREK-1 is a heat-activated background $\mathrm{K}+$ channel. EMBO / 2000; 19: 2483-91.

Mazella J, Zsurger N, Navarro V, et al. The 100-kDa neurotensin receptor is gp95/ sortilin, a non-G-protein-coupled receptor. J Biol Chem 1998; 273: 26273-6.

Mazella J, Petrault O, Lucas G, et al. Spadin, a sortilin-derived peptide, targeting rodent TREK-1 channels: a new concept in the antidepressant drug design. PLoS Biol 2010; 8: e1000355.

Munck Petersen C, Nielsen MS, Jacobsen C, et al. Propeptide cleavage conditions sortilin/ neurotensin receptor-3 for ligand binding. $E M B O$ / 1999; 18: 595-604.

Nestler EJ, Barrot M, DiLeone RJ, et al. Neurobiology of depression. Neuron 2002; 34: 13-25.

Nibuya M, Nestler EJ, Duman RS. Chronic antidepressant administration increases the expression of CAMP response element binding protein (CREB) in rat hippocampus. J Neurosci 1996; 16: 2365-72.

Nielsen MS, Madsen P, Christensen EI, et al. The sortilin cytoplasmic tail conveys Golgiendosome transport and binds the VHS domain of the GGA2 sorting protein. EMBO J 2001; 20: 2180-90.

Noel J, Zimmermann K, Busserolles J, et al. The mechano-activated $\mathrm{K}+$ channels TRAAK and TREK-1 control both warm and cold perception. EMBO / 2009; 28: 1308-18.

Nykjaer A, Lee R, Teng KK, et al. Sortilin is essential for proNGF-induced neuronal cell death. Nature 2004; 427: 843-8.

Perlis RH, Moorjani P, Fagerness J, et al. Pharmacogenetic Analysis of Genes Implicated in Rodent Models of Antidepressant Response: Association of TREK1 and Treatment Resistance in the $\operatorname{STAR}\left({ }^{*}\right) \mathrm{D}$ Study. Neuropsychopharmacology 2008; 33: 2810-9.

Santarelli L, Saxe M, Gross C, et al. Requirement of hippocampal neurogenesis for the behavioral effects of antidepressants. Science 2003; 301: 805-9.

Westergaard UB, Sorensen ES, Hermey G, et al. Functional organization of the sortilin Vps10p domain. J Biol Chem 2004; 279: 50221-9. 